\title{
Alcaligenes is commensal bacteria habituating in the gut-associated lymphoid tissue for the regulation of intestinal IgA responses
}

\author{
Jun Kunisawa ${ }^{1,2}$ * and Hiroshi Kiyono ${ }^{1,2,3,4}$ * \\ 1 Division of Mucosal Immunology, Department of Microbiology and Immunology, Institute of Medical Science, The University of Tokyo, Tokyo, Japan \\ 2 Department of Medical Genome Science, Graduate School of Frontier Science, The University of Tokyo, Tokyo, Japan \\ ${ }^{3}$ Graduate School of Medicine, The University of Tokyo, Tokyo, Japan \\ ${ }^{4}$ Core Research for Evolutional Science and Technology, Japan Science and Technology Agency, Tokyo, Japan
}

\section{Edited by:}

Nils Yngve Lycke, University of Gothenburg, Sweden

\section{Reviewed by:}

Paul King, Monash University, Australia

Hiroshi Ohno, RIKEN, Japan

\section{*Correspondence:}

Jun Kunisawa and Hiroshi Kiyono,

Division of Mucosal Immunology, Department of Microbiology and Immunology, Institute of Medical Science, The University of Tokyo,

Tokyo, Japan.

e-mail: kunisawa@ims.u-tokyo.ac.jp;

kiyono@ims.u-tokyo.ac.jp
Secretory-immunoglobulin A (S-lgA) plays an important role in immunological defense in the intestine. It has been known for a long time that microbial stimulation is required for the development and maintenance of intestinal IgA production. Recent advances in genomic technology have made it possible to detect uncultivable commensal bacteria in the intestine and identify key bacteria in the regulation of innate and acquired mucosal immune responses. In this review, we focus on the immunological function of Peyer's patches (PPs), a major gut-associated lymphoid tissue, in the induction of intestinal IgA responses and the unique immunological interaction of PPs with commensal bacteria, especially Alcaligenes, a unique indigenous bacteria habituating inside PPs.

\section{Keywords: Peyer's patch, IgA, commensal bacteria}

\section{INTRODUCTION}

Secretory-immunoglobulin A (S-IgA) is predominantly observed in the intestine where it participates in immune defense (Mestecky et al., 2005; Brandtzaeg, 2010). S-IgA inhibits adherence of pathogens to host epithelial cells in the intestinal lumen and neutralizes pathogenic toxins by binding to the toxins' biologically active sites. Based on the immunological importance of S-IgA in immunosurveillance in the intestine, the development of oral vaccines has focused on the induction of antigen-specific S-IgA responses (Kunisawa et al., 2007). In addition to the immunosurveillance in the intestine, S-IgA antibody contributes to the establishment of beneficial gut commensal microbiota and thus dysfunction of S-IgA formation resulted in the alteration of normal bacterial flora (e.g., the reduction of Lactobacillus and increase of segmented filamentous bacteria, SFB; Suzuki et al., 2004).

Peyer's patches (PPs) are major gut-associated lymphoid tissue (GALT) where intestinal IgA responses are initiated and regulated by unique immunological crosstalk via cytokines [e.g., interleukin4 (IL-4), IL-6, IL-21, and transforming growth factor- $\beta$ (TGF- $\beta$ )] and cell-cell interactions (e.g., via CD40/CD40 ligand interactions) among dendritic, T, and B cells (Kunisawa et al., 2008; Fagarasan et al., 2010). Thus, oral delivery of antigens to PPs is considered an important strategy for the effective induction of antigen-specific intestinal IgA responses (Kunisawa et al., 2011).

In addition to host-derived factors, microbial stimulation is also required for the maximum production of S-IgA in the intestine (Cebra et al., 2005). Indeed, germ-free (GF) mice have decreased intestinal IgA responses with immature structure of GALT when compared with mice housed under SPF or conventional conditions
(Weinstein and Cebra, 1991). Although it was reported that some commensal bacteria [e.g., SFB and altered Schaedler flora (ASF), a combined eight culturable bacteria] and bacterial products (e.g., peptidoglycan, CpG oligonucleotide, and LPS) stimulated the intestinal IgA production (Michalek et al., 1983; Talham et al., 1999; Butler et al., 2005), it is obscure which bacteria is involved in this process indigenously. Because predominant commensal bacteria in the intestine is uncultivable, it was difficult to determine by culture-based method which bacteria regulated specific immune responses. However, recent advances in the genomic analysis allowed us to identify the uncultivable bacteria, which revealed key bacteria in the regulation of specific immune responses (Ivanov et al., 2009; Atarashi et al., 2011) as well as the development of immune diseases (Chow et al., 2010; Hill and Artis, 2010). Using genomic and immunological methods, we recently found that the microbial community inside PPs is different from those on the epithelium of PPs or in the intestinal lumen (Obata et al., 2010).

In this review, we discuss initially the immunological features of PPs in the induction and regulation of intestinal IgA responses. In the later part, we focus on the unique crosscommunication between PPs and habitat commensal bacteria, Alcaligenes, a unique indigenous bacteria habituating inside PPs and regulating dendritic cells (DCs) for the efficient production of intestinal IgA.

\section{IMMUNOLOGICAL FEATURES OF PEYER'S PATCHES}

In the intestine, GALT comprise several different, organized lymphoid structures (Spencer et al., 2009; Fagarasan et al., 2010). Among them, PPs are the largest and most well-characterized sites 
for the initiation of intestinal IgA responses, especially responses to T cell-dependent antigens (Kunisawa et al., 2008; Fagarasan et al., 2010). There are generally 8-10 PPs in the mouse small intestine and hundreds in the human small intestine. Each PP is composed of several B cell-rich follicles surrounded by a mesh-like structure consisting of T cells known as the interfollicular region (Figure 1).

Inside PPs, antigen-sampling $M$ cells located in the follicleassociated epithelium transport luminal antigens to DCs situated in the subepithelium region (Neutra et al., 2001), which then form clusters with T-, B-, and stromal cells in the germinal centers and promote $\mu$-to- $\alpha$-class-switch recombination of B cells with the help of cytokines such as IL-4, IL-21, and TGF- $\beta$ (Fagarasan et al., 2010). Upon immunoglobulin class-switching from $\mu$ to $\alpha$, IgA-committed B cells ( $\operatorname{IgA}^{+} \mathrm{B}$ cells) begin to express type 1 sphingosine-1-phosphate receptor, CCR9, and $\alpha 4 \beta 7$ integrin, allowing them to depart from the PPs and subsequently traffic to the intestinal lamina propria (Mora et al., 2006; Gohda et al., 2008). In the intestinal lamina propria, they further differentiate into IgA-secreting plasma cells under the influence of terminal differentiation factors (e.g., IL-6; Cerutti et al., 2011). DCs play a key role in these processes. For instance, nitric oxide, TGF- $\beta$, APRIL, and BAFF produced by TNF- $\alpha /$ iNOS-producing DCs (Tip-DCs) promotes IgA production (Tezuka et al., 2007). Also, DCs in the PPs metabolite vitamin A and produce retinoic acid, which induces the expression of gut-homing receptors (CCR9, and $\alpha 4 \beta 7$ integrin) on activated B and T cells (Iwata et al., 2004; Mora et al., 2006). Retinoic acid also induces the preferential differentiation into regulatory $\mathrm{T}$ (Treg) cells (Hall et al., 2011), and some of Treg cells differentiated into follicular helper $\mathrm{T}$ cells to promote IgA production in the PPs (Tsuji et al., 2009).

The identification of the molecular pathway of PP organogenesis allowed the establishment of PP-deficient mice through the loss of any part of this pathway (Nishikawa et al., 2003). Notably, disruption of the PP organogenesis pathway by blockade of tissue genesis cytokine receptor signaling [IL-7R and/or lymphotoxin$\beta$ receptor (LT $\beta R$ )] during a limited fetus time period results in the selective loss of PPs without affecting other lymphoid

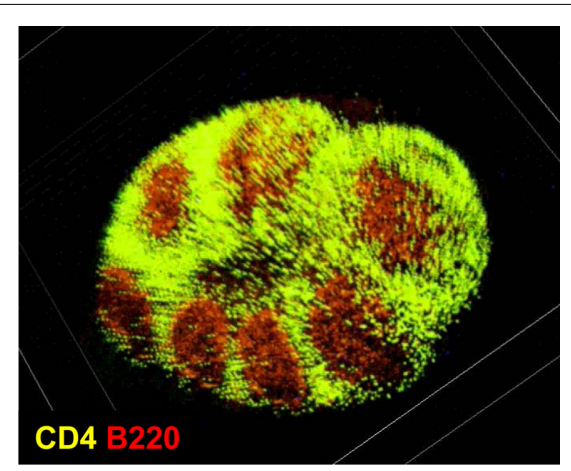

FIGURE 1 | Microarchitecture of murine Peyer's patches. Purified T cells (green) and B cells (red) were chemically labeled with carboxyfluorescein succinimidyl ester and carboxy-SNARF-1, respectively, and adoptively transferred into mice. Fifteen hours after the transfer, cell distribution in the Peyer's patches was observed at the whole-tissue level by using macro-confocal microscopy. tissue organogenesis (Yoshida et al., 1999). Experiments with PPdeficient mice showed that the dependency on PPs in the induction of antigen-specific IgA responses depends on the form of the antigen. For instance, the PP-deficient mice failed to develop antigen-specific IgA responses against orally administered antigens in particle form, but retained their ability to respond to soluble forms of antigens (Yamamoto et al., 2000; Kunisawa et al., 2002). It was also reported that lamina propria DCs are capable of initiating systemic IgG responses, whereas antigen transport by $\mathrm{M}$ cells into the PPs is required for the induction of intestinal IgA production (Martinoli et al., 2007). This is consistent with another finding that DCs in the PPs are responsible for intestinal IgA production (Fleeton et al., 2004). Therefore, PPs are considered to be one of the major sites for the initiation of intestinal antigen-specific IgA responses.

\section{EFFECT OF MICROBIAL STIMULATION ON THE PRODUCTION OF INTESTINAL IgA}

It is well known that microbial stimulation is required for the full production of S-IgA in the intestine. Indeed, GF mice have decreased intestinal IgA responses when compared with mice housed under SPF or conventional conditions (Cebra et al., 2005). Studies using mono-associated GF mice with SFB have demonstrated that only a minor proportion of the total intestinal IgA is reactive to mono-associated bacteria (Talham et al., 1999). In addition, bacterial products produced by commonly expressed on commensal bacteria (e.g., peptidoglycan, CpG oligonucleotide, and LPS) stimulated the intestinal IgA production (Michalek et al., 1983; Butler et al., 2005). In contrast, a recent study using reversible colonization of GF mice with genetically engineered $E$. coli showed that intestinal IgA induced in those mice bound to parent strain but not other bacteria (Hapfelmeier et al., 2010). Therefore, it remains unclear whether intestinal IgA responses induced by commensal bacteria is mediated by polyclonal stimulation and/or by $B$ cell receptors specific for microbial antigens.

As one mechanism of impaired IgA production of GF mice, it was reported that GF mice have structurally immature GALT (e.g., PPs and ILFs) when compared with SPF mice (Weinstein and Cebra, 1991; Hamada et al., 2002). In the PPs, several key pathways for the IgA production require microbial stimulation. For example, Tip-DCs enhance the IgA production by producing nitric oxide, TGF- $\beta$, APRIL, and BAFF, which requires microbial stimulation through innate receptors (Tezuka et al., 2007). Indeed, the number of Tip-DCs was much reduced in the intestine of GF and MyD88-deficient mice (Tezuka et al., 2007). Another cell involved the microbe-dependent IgA production is non-hematopoietic follicular DCs (FDCs). It was reported that microbial stimulation of FDCs resulted in expressing chemokine CXCL13, BAFF, and TGF$\beta$ for the germinal center formation and $B$ cell class-switching from IgM to IgA (Suzuki et al., 2010).

\section{ALCALIGENES IS A UNIQUE INDIGENOUS BACTERIA INSIDE PPs}

Recent advances in genomic technology make it possible to detect commensal bacteria in the intestine, allowing identification of key bacteria involved in the regulation of specific immune responses. For example, SFB was identified as commensal bacteria inducing 
Th17 cells (Ivanov et al., 2009), whereas colonic regulatory T cells were induced by Clostridium clusters IV and XIV (Atarashi et al., 2011). These commensal bacteria localize at the surface of intestinal epithelium, but we supposed that the immunological crosstalk between host and commensal bacteria might establish in the regulation of intestinal IgA responses in the GALT. In this issue, we analyzed the composition of the microbial community inside PPs and identified Alcaligenes as a major commensal bacteria uniquely locating inside PPs (Obata et al., 2010).

By using the 16S rRNA clone library method, SFB are the predominant commensal bacteria co-habitat on FAE of PPs as like small intestinal epithelium. Although the FAE consisted with antigen-sampling $M$ cells, SFB was not found inside of PPs. Instead, Alcaligenes are predominant bacteria inside PPs. The result obtained by the 16S rRNA analysis was further confirmed by fluorescence in situ hybridization (FISH) method and thus Alcaligenes are present exclusively inside PPs, not on the FAE of PPs, and intestinal villous epithelium and intestinal lamina propria (Figure 2). Of note, the preferential presence of Alcaligenes was observed not only in mouse but also in monkey and human (Obata et al., 2010). One of interesting but unresolved questions is the species specificity of Alcaligenes. We are now investigating whether Alcaligenes isolated from human or monkey colonize in the PPs to promote IgA production when they are orally fed to GF mice. Inside PPs, a proportion of the Alcaligenes seemed to be alive in mice. The presence and growth of Alcaligenes were detected in the PPs of GF mice after adoptive transfer of PP homogenates containing Alcaligenes from SPF mice. These findings suggest that Alcaligenes are indigenous bacteria ubiquitously living inside the $\mathrm{PPs}$ of various mammalian species.

\section{ANTIBODY-MEDIATED RECIPROCAL INTERACTION BETWEEN ALCALIGENES AND THE HOST IMMUNE SYSTEM} As mentioned above, $M$ cells located on the FAE of PPs transport luminal bacteria into DCs locating at the subepithelial region of FAE (Neutra et al., 2001). 16S rRNA clone library methods

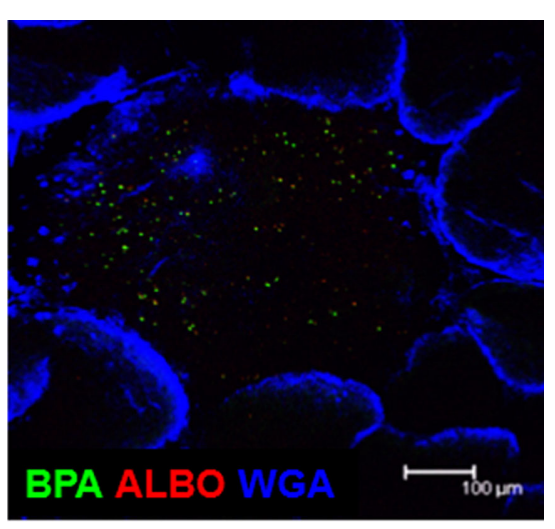

FIGURE 2 | Microarchitecture of murine Peyer's patches. Whole-mount fluorescence in situ hybridization was performed to visually analyze the presence of Alcaligenes inside PPs. Both BPA and ALBO34a were used as specific probes for Alcaligenes. Wheat germ agglutinin (WGA), an $\mathrm{N}$-acetylglucosamine-specific lectin, was used to detect epithelial cells. Scale bar indicates $100 \mu \mathrm{m}$. consistently revealed that DCs in the PPs predominantly contain Alcaligenes, whereas these bacteria are rarely detected in DCs isolated from other lymphoid tissues (e.g., spleen and mesenteric lymph nodes; Obata et al., 2010). We examined the immunological effects of Alcaligenes on DCs and found that the production of IgA-enhancing cytokines such as IL- 6 , TGF- $\beta$, and BAFF was increased when DCs isolated from the PPs of GF mice were stimulated with Alcaligenes (Obata et al., 2010). Several lines of evidence have revealed that immunological functions of DCs are different between intestinal and other lymphoid tissues (reviewed in Rescigno, 2010), we are now investigating whether immune stimulatory functions of Alcaligenes is specific for the PP DCs or not.

In agreement with the uptake of Alcaligenes and subsequent production of IgA-enhancing cytokines by DCs, Alcaligenesspecific IgA-forming cells were frequently observed in PPs, and consequent IgA production was noted in the intestinal lumen of SPF mice, but not GF mice (Obata et al., 2010). Although biological role of Alcaligenes-specific IgA antibody remains to be elucidated, the antibody might be involved in the creation of intratissue co-habitation of Alcaligenes in PPs. To this end, the number of Alcaligenes inside PPs is decreased in B cell-deficient CBA/N xid and IgA-deficient mice compared with wild-type mice (Obata et al., 2010). Therefore, it is interesting to suggest that Alcaligenes-specific IgA antibody mediates the uptake and presence of Alcaligenes in the PPs. Since M cells express IgA receptors

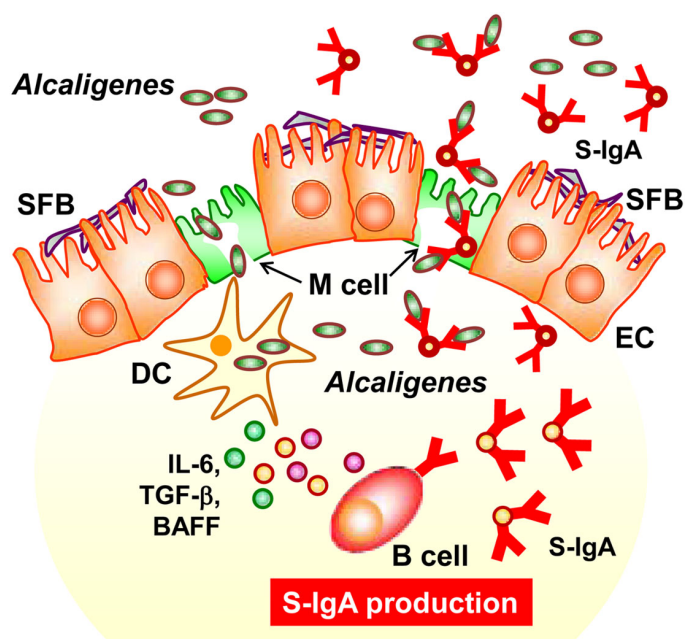

Peyer's patch

FIGURE 3 | Alcaligenes mediates symbiotic communication inside Peyer's patches. On the follicle-associated epithelium of PPs, segmented filamentous bacteria (SFB) is predominantly observed. In contrast, Alcaligenes specifically localizes inside Peyer's patches, where some are taken up by dendritic cells (DCs). Stimulation by Alcaligenes prompts the DCs to produce IgA-enhancing cytokines [e.g., interleukin-6 (IL-6), transforming growth factor- $\beta$ (TGF- $\beta$ )], and B cell activating factor (BAFF), which enhance the intestinal $\lg A$ response. The intestinal $\lg A$ includes Alcaligenes-specific $\lg A$, which might mediate the preferential uptake and presence of Alcaligenes in the PPs. The uptake is presumably mediated by $\mathrm{M}$ cells. 
(Mantis et al., 2002), one possibility is that Alcaligenes coated with the Alcaligenes-specific antibody are taken up into PPs through $\mathrm{M}$ cells. Further, the antigen-specific IgA coating on Alcaligenes might be beneficial for the bacteria to create the co-habitation niche since IgA antibody has been shown to non-inflammatory antibody (Mestecky et al., 2005).

\section{CONCLUSION}

In this review, we discussed a new concept of symbiotic communication in PPs that is mediated by commensal bacteria-specific IgA antibody. Alcaligenes-specific antibodies may mediate the uptake and the presence of Alcaligenes in the PPs, and the co-habitation of Alcaligenes within the PPs is one of the key factors to promote the intestinal IgA production by enhancing the production of IgA-enhancing cytokines from DCs (Figure 3). We still have various questions regarding this co-habitation of Alcaligenes in the PPs. For example, it remains unclear whether the presence of Alcaligenes inside of PPs is physiologically beneficial or harmful for the host immune system. In this issue, we are now addressing the microbial community in the PPs of mice and human patients suffering from intestinal immune diseases (e.g., intestinal inflammation and allergy). The biological roles of intra-tissue habitation of Alcaligenes in the PPs in the appropriate regulation of mucosal immune responses need to be elucidated. The current goal is to elucidate the mechanisms behind the co-habitation of Alcaligenes within PPs, and the exact contribution of Alcaligenes to educate and guide mucosal immunocompetent cells especially

\section{REFERENCES}

Atarashi, K., Tanoue, T., Shima, T., Imaoka, A., Kuwahara, T., Momose, Y., Cheng, G., Yamasaki, S., Saito, T., Ohba, Y., Taniguchi, T., Takeda, K., Hori, S., Ivanov, I. I., Umesaki, Y., Itoh, K., and Honda, K. (2011). Induction of colonic regulatory $\mathrm{T}$ cells by indigenous Clostridium species. Science 331, 337-341.

Brandtzaeg, P. (2010). Function of mucosa-associated lymphoid tissue in antibody formation. Immunol. Invest. 39, 303-355.

Butler, J. E., Francis, D. H., Freeling, J., Weber, P., and Krieg, A. M. (2005). Antibody repertoire development in fetal and neonatal piglets. IX. Three pathogen-associated molecular patterns act synergistically to allow germfree piglets to respond to type 2 thymus-independent and thymusdependent antigens. J. Immunol. $175,6772-6785$.

Cebra, J. J., Jiang, H. Q., Boiko, N. V., and Tlaskalva-Hogenova, H. (2005). "The role of mucosal microbiota in the development, maintenance, and pathologies of the mucosal immune system," in Mucosal Immunology, 3rd Edn, eds J. Mestecky, M. E. Lamm, W. Strober, J. Bienenstock, J. R. Mcghee, and L. Mayer (San Diego: Academic Press), 335-368.
Cerutti, A., Chen, K., and Chorny, A. (2011). Immunoglobulin responses at the mucosal interface. Annu. Rev. Immunol. 29, 273-293.

Chow, J., Lee, S. M., Shen, Y., Khosravi, A., and Mazmanian, S. K. (2010). Host-bacterial symbiosis in health and disease. Adv. Immunol. 107, 243-274.

Fagarasan, S., Kawamoto, S., Kanagawa, O., and Suzuki, K. (2010). Adaptive immune regulation in the gut: $T$ celldependent and $\mathrm{T}$ cell-independent IgA synthesis. Annu. Rev. Immunol. $28,243-273$.

Fleeton, M. N., Contractor, N., Leon, F., Wetzel, J. D., Dermody, T. S., and Kelsall, B. L. (2004). Peyer's patch dendritic cells process viral antigen from apoptotic epithelial cells in the intestine of reovirusinfected mice. J. Exp. Med. 200, 235-245.

Gohda, M., Kunisawa, J., Miura, F., Kagiyama, Y., Kurashima, Y., Higuchi, M., Ishikawa, I., Ogahara, I., and Kiyono, H. (2008). Sphingosine 1-phosphate regulates the egress of IgA plasmablasts from Peyer's patches for intestinal IgA responses. J. Immunol. 180, 5335-5343.

Hall, J. A., Grainger, J. R., Spencer, S. P., and Belkaid, Y. (2011). The role of retinoic acid in tolerance and immunity. Immunity 35, 13-22.

DCs in the PPs for the development, maturation and maintenance of the appropriate host immune system. These studies will provide novel molecular and cellular mechanisms of symbiotic communication with commensal bacteria in the regulation of host immunity.

\section{ACKNOWLEDGMENTS}

We thank Dr. T. Obata for his contribution to all works related to Alcaligenes, Drs. Y. Benno and M. Sakamoto (RIKEN BioResource Center), and Drs. Y. Umesaki, T. Matsuki, H. Setoyama (Yakult Central Institute for Microbiological Research) for their constructive discussions, technical advice, and supports on microbial analyses, and Dr. H. Iijima (Osaka University) for providing human samples. The work related to this review was supported by grants from the Ministry of Education, Culture, Sports, Science and Technology of Japan [Grant-in-Aid for Young Scientists A (22689015 to Jun Kunisawa), for Scientific Research on Innovative Areas (23116506 to Jun Kunisawa), for Scientific Research S (23229004 to Hiroshi Kiyono), and for Scientific Research on Priority Area (19059003 to Hiroshi Kiyono)]; the Ministry of Health and Welfare of Japan (to Jun Kunisawa and Hiroshi Kiyono); the Global Center of Excellence Program of the Center of Education and Research for Advanced Genome-based Medicine (to Hiroshi Kiyono); the Program for Promotion of Basic and Applied Researches for Innovations in Bio-oriented Industry (to Jun Kunisawa); and the Yakult Bio-Science Foundation (to Jun Kunisawa).

Hamada, H., Hiroi, T., Nishiyama, Y., Takahashi, H., Masunaga, Y., Hachimura, S., Kaminogawa, S., Takahashi-Iwanaga, H., Iwanaga, T. Kiyono, H., Yamamoto, H., and Ishikawa, H. (2002). Identification of multiple isolated lymphoid follicles on the antimesenteric wall of the mouse small intestine. J. Immunol. 168, 57-64.

Hapfelmeier, S., Lawson, M. A., Slack, E., Kirundi, J. K., Stoel, M., Heikenwalder, M., Cahenzli, J., Velykoredko, Y., Balmer, M. L., Endt, K., Geuking, M. B., Curtiss, R. III, Mccoy, K. D., and Macpherson, A. J. (2010). Reversible microbial colonization of germ-free mice reveals the dynamics of IgA immune responses. Science 328, 1705-1709.

Hill, D. A., and Artis, D. (2010). Intestinal bacteria and the regulation of immune cell homeostasis. Annu. Rev. Immunol. 28 623-667.

Ivanov, I. I., Atarashi, K., Manel, N., Brodie, E. L., Shima, T., Karaoz, U., Wei, D., Goldfarb, K. C., Santee, C. A., Lynch, S. V., Tanoue, T., Imaoka, A., Itoh, K., Takeda, K., Umesaki, Y., Honda, K., and Littman, D. R. (2009). Induction of intestinal Th17 cells by segmented filamentous bacteria. Cell 139, 485-498.
Iwata, M., Hirakiyama, A., Eshima, Y., Kagechika, H., Kato, C., and Song, S. Y. (2004). Retinoic acid imprints gut-homing specificity on $\mathrm{T}$ cells. Immunity 21, 527-538.

Kunisawa, J., Kurashima, Y., and Kiyono, H. (2011). Gut-associated lymphoid tissues for the development of oral vaccines. Adv. Drug Deliv. Rev. [Epub ahead of print].

Kunisawa, J., Mcghee, J., and Kiyono, H. (2007). "Mucosal S-IgA enhancement: development of safe and effective mucosal adjuvants and mucosal antigen delivery vehicles," in Mucosal Immune Defense: Immunoglobulin $A$, ed. C. Kaetzel (New York: Kluwer Academic/Plenum Publishers), 346-389.

Kunisawa, J., Nochi, T., and Kiyono, H. (2008). Immunological commonalities and distinctions between airway and digestive immunity. Trends Immunol. 29, 505-513.

Kunisawa, J., Takahashi, I., Okudaira, A., Hiroi, T., Katayama, K., Ariyama, T., Tsutsumi, Y., Nakagawa, S., Kiyono, H., and Mayumi, T. (2002). Lack of antigen-specific immune responses in anti-IL-7 receptor $\alpha$ chain antibody-treated Peyer's patch-null mice following intestinal immunization with microencapsulated antigen. Eur. J. Immunol. 32, 2347-2355. 
Mantis, N. J., Cheung, M. C., Chintalacharuvu, K. R., Rey, J., Corthesy, B., and Neutra, M. R. (2002). Selective adherence of IgA to murine Peyer's patch M cells: evidence for a novel IgA receptor. J. Immunol. 169, 1844-1851.

Martinoli, C., Chiavelli, A., and Rescigno, M. (2007). Entry route of Salmonella typhimurium directs the type of induced immune response. Immunity 27, 975-984.

Mestecky, J., Moro, I., Kerr, M. A., and Woof, J. M. (2005). "Mucosal immunoglobulins," in Mucosal Immunology, 3rd Edn, eds J. Mestecky, M. E. Lamm, W. Strober, J. Bienenstock, J. R. Mcghee, and L. Mayer (San Diego: Academic Press), 153-182.

Michalek, S. M., Morisaki, I., Gregory, R. L., Kiyono, H., Hamada, S., and Mcghee, J. R. (1983). Oral adjuvants enhance IgA responses to Streptococcus mutans. Mol. Immunol. 20, 1009-1018.

Mora, J. R., Iwata, M., Eksteen, B., Song, S. Y., Junt, T., Senman, B., Otipoby, K. L., Yokota, A., Takeuchi, H., Ricciardi-Castagnoli, P., Rajewsky, K., Adams, D. H., and Von Andrian, U. H. (2006). Generation of gut-homing IgA-secreting B cells by intestinal dendritic cells. Science 314, 1157-1160.

Neutra, M. R., Mantis, N. J., and Kraehenbuhl, J. P. (2001). Collaboration of epithelial cells with organized mucosal lymphoid tissues. Nat. Immunol. 2, 1004-1009.

Nishikawa, S., Honda, K., Vieira, P., and Yoshida, H. (2003). Organogenesis of peripheral lymphoid organs. Immunol. Rev. 195, 72-80.

Obata, T., Goto, Y., Kunisawa, J., Sato, S., Sakamoto, M., Setoyama, H., Matsuki, T., Nonaka, K., Shibata, N., Gohda, M., Kagiyama, Y., Nochi, T., Yuki, Y., Fukuyama, Y., Mukai, A., Shinzaki, S., Fujihashi, K., Sasakawa, C., Iijima, H., Goto M., Umesaki, Y., Benno, Y., and Kiyono, H. (2010). Indigenous opportunistic bacteria inhabit mammalian gut-associated lymphoid tissues and share a mucosal antibody-mediated symbiosis. Proc. Natl. Acad. Sci. U.S.A. 107, 7419-7424.

Rescigno, M. (2010). Intestinal dendritic cells. Adv. Immunol. 107, 109-138.

Spencer, J., Barone, F., and DunnWalters, D. (2009). Generation of immunoglobulin diversity in human gut-associated lymphoid tissue. Semin. Immunol. 21, 139-146.

Suzuki, K., Maruya, M., Kawamoto, S., Sitnik, K., Kitamura, H., Agace, W. W., and Fagarasan, S. (2010). The sensing of environmental stimuli by follicular dendritic cells promotes immunoglobulin A generation in the gut. Immunity 33, 71-83.

Suzuki, K., Meek, B., Doi, Y., Muramatsu, M., Chiba, T., Honjo, T., and Fagarasan, S. (2004). Aberrant expansion of segmented filamentous bacteria in IgA-deficient gut. Proc. Natl. Acad. Sci. U.S.A. 101, 1981-1986.

Talham, G. L., Jiang, H. Q., Bos, N. A., and Cebra, J. J. (1999). Segmented filamentous bacteria are potent stimuli of a physiologically normal state of the murine gut mucosal immune system. Infect. Immun. 67, 1992-2000.

Tezuka, H., Abe, Y., Iwata, M., Takeuchi, H., Ishikawa, H., Matsushita, M., Shiohara, T., Akira, S., and Ohteki, T. (2007). Regulation of IgA production by naturally occurring TNF/iNOS-producing dendritic cells. Nature 448, 929-933.

Tsuji, M., Komatsu, N., Kawamoto, S. Suzuki, K., Kanagawa, O., Honjo, T., Hori, S., and Fagarasan, S. (2009). Preferential generation of follicular $\mathrm{B}$ helper $\mathrm{T}$ cells from Foxp3+ $\mathrm{T}$ cells in gut Peyer's patches. Science 323, 1488-1492.

Weinstein, P. D., and Cebra, J. J. (1991). The preference for switching to IgA expression by Peyer's patch germinal center B cells is likely due to the intrinsic influence of their microenvironment. J. Immunol. 147, 4126-4135.

Yamamoto, M., Rennert, P., Mcghee, J. R., Kweon, M. N., Yamamoto, S., Dohi, T., Otake, S., Bluethmann, H., Fujihashi, K., and Kiyono, H. (2000). Alternate mucosal immune system: organized Peyer's patches are not required for IgA responses in the gastrointestinal tract. J. Immunol. 164, 5184-5191.

Yoshida, H., Honda, K., Shinkura, R. Adachi, S., Nishikawa, S., Maki, K., Ikuta, K., and Nishikawa, S. I. (1999). IL-7 receptor $\alpha^{+}$CD3- cells in the embryonic intestine induces the organizing center of Peyer's patches. Int. Immunol. 11, 643-655.

Conflict of Interest Statement: The authors declare that the research was conducted in the absence of any commercial or financial relationships that could be construed as a potential conflict of interest.

Received: 27 January 2012; paper pending published: 15 February 2012; accepted: 15 March 2012; published online: 02 April 2012.

Citation: Kunisawa $J$ and Kiyono $H$ (2012) Alcaligenes is commensal bacteria habituating in the gut-associated lymphoid tissue for the regulation of intestinal IgA responses. Front. Immun. 3:65. doi: 10.3389/fimmu.2012.00065

This article was submitted to Frontiers in Mucosal Immunity, a specialty of Frontiers in Immunology.

Copyright () 2012 Kunisawa and Kiyono. This is an open-access article distributed under the terms of the Creative Commons Attribution Non Commercial License, which permits non-commercial use, distribution, and reproduction in other forums, provided the original authors and source are credited. 